\title{
Mutation and expression of multiple treatment response-related genes in a population with locally advanced non-small cell lung cancer
}

\author{
HONG-YANG LU ${ }^{1}$, DAN SU ${ }^{1}$, XIAO-DAN PAN ${ }^{1}$, HONG JIANG ${ }^{1}$ and SHENG-LIN MA ${ }^{2}$ \\ ${ }^{1}$ Zhejiang Key Laboratory of the Diagnosis and Treatment Technology on Thoracic Oncology, Zhejiang Cancer Hospital, \\ Hangzhou 310022; ${ }^{2}$ Department of Oncology, Hangzhou First People's Hospital, Hangzhou 310006, P.R. China
}

Received August 23, 2011; Accepted November 15, 2011

DOI: $10.3892 / \mathrm{ol} .2011 .502$

\begin{abstract}
Individual therapy based on various pathohistological types and biological characteristics may be the practical trend of advanced non-small cell lung cancer (NSCLC) treatment. To provide a molecular criterion for drug selection, we investigated the incidence of somatic mutation and mRNA expression levels of common genes relevant to treatment response in a population with locally advanced NSCLC. Mutant-enriched and branched DNA-liquidchip technology (bDNA-LCT) were used to detect the somatic mutations in the epidermal growth factor receptor (EGFR), KRAS, BRAF and phosphatidylinositol-3-kinase catalytic $\alpha$ (PIK3CA) genes, and mRNA levels of EGFR, ERCC1, class III $\beta$-tubulin (TUBB3) and TYMS, separately, in paraffin tissue blocks from 30 patients with stage IIIA NSCLC. Our current findings revealed that 6,4 and 2 out of 30 samples were found with mutations in exons 19, 21 and 20 of the EGFR gene, respectively. The mutation incidence of exons 19 and 21 had a positive correlation with EGFR mRNA expression. Mutations in exons 12 and 13 of the K-ras gene were found in 2 out of 30 , and 1 out of 30 samples, separately. Three out of 30 samples were found with mutations in codon 542 of the PIK3CA gene. No mutations were found in the BRAF gene. The expression levels of ERCC1 and TUBB3 mRNAs were higher in patients with adenocarcinoma than those in patients with squamous cell carcinoma. The expression of TYMS mRNA in patients with adenocarcinoma was lower than that in patients with
\end{abstract}

Correspondence to: Professor Sheng-Lin Ma, Hangzhou First People's Hospital, 261 Huan Sha Road, Hangzhou 310006, P.R. China E-mail: shenglinma@163.com

Abbreviations: EGFR, epidermal growth factor receptor; BRAF, serine/threonine-protein kinase; PIK3CA, phosphoinositide-3-kinase; ERCC1, excision repair cross-complementing rodent repair deficiency, complementation group 1; TUBB3, tubulin, $\beta 3$; TYMS, thymidylate synthase; NSCLC, non-small cell lung cancer; FFPE, formalin-fixed paraffin-embedded

Key words: gene mutation, expression, treatment response related genes, locally advanced non-small cell lung cancer squamous cell carcinoma. In conclusion, mutations and mRNA expression of these commonly studied genes provides a basis for the selection of suitable molecular markers for individual treatment in a population with locally advanced NSCLC.

\section{Introduction}

Patients with locally advanced non-small cell lung cancer (LA-NSCLC), which cannot be resected, account for one third of non-small cell lung cancer (NSCLC) cases. Although synchronic radio-chemotherapy is the traditional cancer treatment, two problems remain. First, increasing the response to radio-chemotherapy corresponds to an increase in adverse effects; second, the survival time of patients receiving synchronic radio-chemotherapy has not yet proved satisfactory (1). Therefore, how to select the optimum regimen for these populations, in order to increase the treatment response and decrease the adverse effects, should be further investigated.

In recent years, mutations in the epidermal growth factor receptor (EGFR) gene have been reported to be present in NSCLC and correlate with the response of EGFR tyrosine kinase inhibitors (TKIs), suggesting their usefulness as a biomarker. The INTEREST) trial (2) suggested that gefitinib may provide a similar overall survival (OS) to docetaxel in patients across a broad range of clinical subgroups, and that EGFR biomarkers, such as mutation status, may additionally identify which patients are likely to gain the greatest progression-free survival (PFS) and overall response rate (ORR) benefits from gefitinib. The Iressa Pan-Asia study (IPASS) (3) demonstrates that gefitinib is superior to carboplatin-paclitaxel as an initial treatment for pulmonary adenocarcinoma among non-smokers or former light smokers in East Asia, and that the presence in the tumor of a mutation of the EGFR gene is a strong predictor of an improved outcome with gefitinib. Thus, at present, the identification of patients who are most likely to derive clinical benefit from EGFR-targeted therapy is crucial.

In addition, somatic mutations in the KRAS gene have been reported to confer drug resistance to EGFR TKIs. Expression of ERCC1, class III $\beta$-tubulin (TUBB3) and TYMS are associated with the response to chemotherapy agents, such as 
Table I. Primer sequences.

\begin{tabular}{|c|c|c|c|}
\hline Name & Site & Sequence & 1 st or 2 nd \\
\hline E19-S1-bio & EGFR Exon 19 & 5'biotin-ATCCCAGAAGAGAAGATAATTC3' & 1 st and 2 nd \\
\hline E19-As1 & EGFR Exon 19 & 5'CCTGAGGTTCAGAGCCATGGA3' & $1 \mathrm{st}$ \\
\hline E19-As2 & EGFR Exon 19 & 5'ACCCCCACAGCAAAGCAG3' & 2 nd \\
\hline E21-S1 & EGFR Exon 21 & 5'CAGCCAGAA ACGTACTGGTGA3' & $1 \mathrm{st}$ \\
\hline E21-As1 & EGFR Exon 21 & 5'TCCGTGGTGTCACGAAAATGCT3' & 1 st and 2 nd \\
\hline $\mathrm{E} 21-\mathrm{S} 2$ & EGFR Exon 21 & 5'biotin-CGCAGCATCTCAGATCACAGAT3' & 2 nd \\
\hline E790F1 & EGFR Exon 20 & 5'GAAGCCTACGTGATGGCCAG3' & $1 \mathrm{st}$ \\
\hline E790R & EGFR Exon 20 & 5'AGCCGAAGGGCATGAGCCGC3' & 1 st and 2 nd \\
\hline E790F2-bio & EGFR Exon 20 & 5'biotin-TACGTGATGGCCAGCGTGGA3' & 2nd \\
\hline K13R-bio & KRAS Codon 13 & 5'biotin-CGTCAAGCCACTCTTGCCGGCG3' & 2 nd \\
\hline $\mathrm{K} 13 \mathrm{~F} 1$ & KRAS Codon 13 & 5'TGGAGTATTTCATAGTGTATTAACC3' & $1 \mathrm{st}$ \\
\hline $\mathrm{K} 13 \mathrm{~F} 2$ & KRAS Codon 13 & 5'TGATAGTGTATTAAGCTTATGTGTG3' & 2nd \\
\hline $\mathrm{B} 15 \mathrm{~F}$ & BRAF Codon 600 & 5'TCATAATGCTTGCTCTGATAGGA3' & 1 st and 2 nd \\
\hline B15R1 & BRAF Codon 600 & 5'TAGCCTCAATTCTTACCATCCAC3' & $1 \mathrm{st}$ \\
\hline B15R2-bio & BRAF Codon 600 & 5'biotin-ACAACTGTTCAACTGATGGGAC3' & 2 nd \\
\hline PE9-AS1 & PI3K Codon 542 & 5'GGAACTTTACACACTGCTGAACC3' & $1 \mathrm{st}$ \\
\hline PE9-S1 & PI3K Codon 542 & 5'GCAGGAGAAAGATTTTCTATGG3' & 1 st and 2 nd \\
\hline PE9-AS2-bio & PI3K Codon 542 & 5'biotin-CTTCTCGGGATACAGACCAAT3' & 2nd \\
\hline
\end{tabular}

cisplatin, paclitaxel and pemetrexed (4-6). Individual therapy based on various pathohistological types and biological characteristics may be the practical trend of LA-NSCLC treatment. However, no relevant studies are currently available reporting the response-related gene mutations and expression status in a Chinese population with LA-NSCLC. To elucidate the incidence of a gene mutation or expression of EGFR, K-Ras, BRAF, phosphatidylinositol-3-kinase catalytic $\alpha$ (PIK3CA), ERCC1, TUBB3 and TYMS in IIIA NSCLC in China, we investigated the gene mutations or mRNA expression levels in paraffin tissue blocks from 30 patients with stage IIIA NSCLC in Zhejiang Cancer Hospital (China), by mutant-enriched and branched DNA-liquidchip technology (bDNA-LCT).

\section{Materials and methods}

Patient characteristics. A total of 30 patients with stage IIIA NSCLC, undergoing treatment in Zhejiang Cancer Hospital between March 2008 and July 2009, were recruited for this study. Patient samples of NSCLC were consecutively collected from the tissue specimen database of Zhejiang Cancer Hospital and all participants had signed informed consent. Use of tissue samples was approved by the Ethical Review Committee of Zhejiang Cancer Hospital. Of the 30 patients, the median age was 58 years (range, 39-74), $22(73.3 \%)$ were male, and 8 $(26.7 \%)$ were female patients. Of the 30 patients, $11(36.7 \%)$ had never smoked, and 19 patients $(63.3 \%)$ had smoked or were still smoking at the time of enrollment. Additionally, $18(60.0 \%)$ patients had adenocarcinoma and $12(40.0 \%)$ had squamous cell carcinoma. Tumor tissues of 6 patients $(20.0 \%)$ were low differentiated, $10(33.3 \%)$ were middle-low differentiated and 13 patients (43.3\%) were middle differentiated, and one was unknown.
Detection of RNA expression levels by bDNA-LCT. Formalin-fixed, paraffin-embedded (FFPE) tissue samples were processed according to the instructions of the manufacturer of the bDNA kit (Panomics, Inc., Fremont, CA, USA). The fluorescence value of each sample was analyzed by the Luminex 200 system (Luminex Co., Austin, TX, USA).

Mutant-enriched liquidchip technology for gene mutation. Mutant-enriched polymerase chain reaction (ME-PCR) is a two-step PCR with intermittent restriction digestion used to selectively eliminate wild-type genes, thus enriching the mutated genes. The ME-PCR method includes three major steps: i) introduction of a restriction site to the wild-type or mutant DNA through PCR amplification to eliminate the wild-type genes by restriction enzyme digestion; ii) selective amplification of the mutated DNA sequence; iii) hybridization of the mutated PCR product to a specific probe, which is precoated on the polystyrene microspheres and analyzed using the Luminex (xMAP) analyzer (Luminex Co.). Using this method, the wild-type DNA is incapable of being completely digested following the first round of PCR. However, following digestion, the ratio of mutant to wild-type DNA was markedly increased. Therefore, the mutant PCR product following the 2nd PCR may be detected using the Luminex analyzer. Assays were performed according to the manufacturers' instructions. For each analyte, 100 beads were analyzed and the median fluorescence intensity was determined.

Primers were designed against the sequences of the target in these genes (Table I). The primers were synthesized by Invitrogen (Shanghai Invitrogen Biotechnology Co., Ltd. China).

To enrich the mutant alleles of these sites, the first PCR amplification was run for 20 cycles $\left(20 \mathrm{sec}\right.$ at $94^{\circ} \mathrm{C}, 30 \mathrm{sec}$ at $58^{\circ} \mathrm{C}$, and $20 \mathrm{sec}$ at $72^{\circ} \mathrm{C}$ ) using Taq DNA polymerase (New 
Table II. EGFR, KRAS, BRAF and PIK3CA mutations detected in non-small cell lung cancer tissues.

\begin{tabular}{lc}
\hline Gene & Mutation, $\mathrm{n}(\%)$ \\
\hline EGFR & \\
Exon 19 & $6(20)$ \\
Exon 21 & $4(13.3)$ \\
Exon 20 & $2(6.7)$ \\
KRAS & \\
Codon 12 & $2(6.7)$ \\
Codon 13 & $1(3.3)$ \\
PIK3CA & \\
Codon 542 & $3(10)$ \\
Codon 545 & 0 \\
Codon 1047 & 0 \\
BRAF & \\
Exon 15 & 0 \\
\hline
\end{tabular}

EGFR, epidermal growth factor receptor; PIK3CA, phosphatidylinositol-3-kinase catalytic $\alpha$.

England Biolabs, Ipswich, MA, USA) and relevant primers. The first primer, which harbored one mismatched site, was used to introduce a new sequence for the wild-type DNA. Following digestion (Promega, Madison, WI, USA), the second PCR was run using the 2 nd primers for 30 cycles $\left(20 \mathrm{sec}\right.$ at $94^{\circ} \mathrm{C}, 30 \mathrm{sec}$ at $60^{\circ} \mathrm{C}$, and $20 \mathrm{sec}$ at $72^{\circ} \mathrm{C}$ ).

In the two types of ME-PCR, the 5' terminus of the reverse primers in the second PCR were labeled with biotin, in order that the PCR product be read by the Luminex.

Statistical analysis. The statistical significance of the mean values was determined by SPSS 13.0. A p-value of $<0.05$ was considered to indicate a statistically significant difference.

A total of 30 patients with stage IIIA NSCLC, undergoing treatment in Zhejiang Cancer Hospital between March 2008 and July 2009, were recruited for this study. Patient samples of NSCLC were consecutively collected using the tissue specimen database of Zhejiang Cancer Hospital and all participants had provided informed consent. The use of tissue samples was approved by the Ethical Review Committee of Zhejiang Cancer Hospital.

\section{Results}

Genetic mutation and expression analysis. The liquid phase array method was used to detect the genetic mutation of EGFR, KRAS, BRAF and PIK3CA, and mRNA expression of EGFR, ERCC1, TUBB3 and TYMS in paraffin waxes from the 30 patients with stage IIIA NSCLC. It was revealed that 6 out of 30 (20\%), 4 out of 30 (13.3\%), and 2 out of 30 samples $(6.9 \%)$ were found with mutations in exons 19, 21 and 20 of the EGFR gene, respectively. Mutations were found in 2 out of $30(6.7 \%)$ and 1 out of 30 samples (3.3\%) in exons 12 and 13 of the K-ras gene. Three out of 30 samples (10\%) were
Table III. The correlation between EGFR active mutation (exons 19 and 21) and clinicopathological factors.

\begin{tabular}{lrc}
\hline Variable & EGFR mutation & P-value \\
\hline Gender & $5 / 22(22.7)$ & \\
Male & $5 / 8(62.5)$ & 0.007 \\
Female & & \\
Smoking & $7 / 11(63.6)$ & 0.041 \\
No & $3 / 19(15.8)$ & \\
Yes & & \\
Histology & $8 / 18(44.4)$ & 0.114 \\
AD & $2 / 12(16.7)$ & \\
SC & & 0.454 \\
Differentiation & $4 / 13(30.8)$ & - \\
Middle & $2 / 10(20.0)$ & \\
Middle-low & $3 / 6(50.0)$ & \\
Low & & \\
\hline
\end{tabular}

EGFR, epidermal growth factor receptor; AD, adenocarcinoma; $\mathrm{SC}$, squamous cell carcinoma.

Table IV. The correlation between EGFR gene mutation and EGFR mRNA expression.

\begin{tabular}{llr}
\hline Variable & $\begin{array}{c}\text { EGFR mRNA } \\
\text { Mean (median, range) }\end{array}$ & P-value \\
\hline EGFR mutation & & \\
Yes & $1.81(1.58,0.18-5.14)$ & 0.043 \\
No & $0.84(0.63,0.00-2.88)$ & \\
\hline
\end{tabular}

EGFR, epidermal growth factor receptor.

found with mutations in codon 542 of PIK3CA. No mutations were detected in the BRAF gene (Table II). Analysis of the correlation between EGFR mutation and clinicopathological factors revealed that mutations in exons 19 and 21 of the EGFR gene were correlated with the gender and smoking status of patients, 5 out of 8 female patients $(62.5 \%)$ were found with mutations in exons 19 or 21 of the EGFR gene, which was much higher than that found in male patients, $22.7 \%(5 / 22)$, $\mathrm{p}=0.007$. A total of 7 out of 11 non-smoking patients $(63.6 \%)$ were found with mutations in exons 19 or 21 of the EGFR gene, which was much higher than in smokers $15.8 \%(3 / 19)$, $\mathrm{p}=0.041$ (Table III). Moreover, mutations in exons 19 or 21 of the EGFR gene had a positive correlation with EGFR mRNA expression, $\mathrm{p}=0.043$ (Table IV).

Expression of ERCC1 mRNA was correlated with gender, histological type and degree of histodifferentiation. The expression of ERCC1 mRNA in female patients with adenocarcinoma was much higher than that found in male patients with squamous cell carcinoma ( $p=0.024$ and $p=0.022$, respectively). In addition, the expression of TUBB3 mRNA in adenocarcinoma patients was much higher than that found in squamous 
cell carcinoma patients, $\mathrm{p}=0.042$; however, the expression of TYMS mRNA in adenocarcinoma patients was lower than that found in squamous cell carcinoma patients, $\mathrm{p}=0.047$ (Table V).

\section{Discussion}

Previous studies have found that mutations are more frequent in females, non-smokers and adenocarcinoma patients $(7,8)$. Our study has reached similar conclusions. At present, we cannot select patients for gefitinib therapy based on clinical factors, such as adenocarcinoma, non-smokers and female gender. Certain adenocarcinoma patients, who are non-smokers and female, may have no effective mutation of EGFR, while certain patients with squamous cell carcinoma, who are smokers and male, may have effective mutations of EGFR; therefore, the mutation condition of EGFR should be detected. Of note is that if we select patients by clinical factors alone, we are likely to treat the patients incorrectly. A correlation between EGFR mutation and gene copy number has been detected. In the present study, we found that mutations of exons 19 or 21 on the EGFR gene, and EGFR mRNA expression had a positive correlation. EGFR mutations were predictive of responses to single-agent TKIs and EGFR gene copy number was also associated with a response to TKIs, albeit with lower sensitivity and specificity (9). Thus, EGFR mutations should be selected, and not EGFR gene copy number gain. The detection of an EGFR mutation is important for gefitinib or erlotinib therapy in advanced NSCLC, particularly in first-line therapy. For the population of LA-NSCLC who require radiotherapy, the response of treatment combined with TKI therapy in LA-NSCLC, with EGFR exons 19 and 21 mutations, should be investigated. The response of 6 months of erlotinib maintenance therapy, following erlotinib combined with a synchronic pectoral radiotherapy of $66 \mathrm{~Gy}$ in 30 patients with LA-NSCLC, was evaluated in a recent clinical trial, and the effective rate in the erlotinib-treated group was found to be higher than the radiotherapy-treated group alone (83 vs. 56\%) (10). At present, a clinical trial is under way with a focus on the evaluation of the response of erlotinib combined with synchronic pectoral radiotherapy in LA-NSCLC patients (RT0901).

The mutation rate of the K-Ras gene in adenocarcinoma patients was 30\%, and the surgical response of this population was poor (11). Results of the present study showed that the mutation rate of the K-Ras gene codon 12 was $6.7 \%$, and codon 13 was $3.3 \%$. Mascaux et al reported that the K-Ras gene mutation was a poor prognosis factor (12). Consistently, the adjuvant cisplatin/vinorelbine subgroup analysis, according to the K-Ras gene mutation, suggested that no apparent benefit was found in patients with K-Ras gene-mutated tumors (13). Moreover, a study by Gautschi et al demonstrated that the prognosis of patients with a K-Ras gene mutation was rather poor, and that tumors with a K-Ras gene mutation were short of sensitivity to gefitinib or erlotinib (14). Conversely, patients with a wild-type K-Ras gene may benefit from TKI therapy (15). However, the results of the INTEREST study (2) revealed that there was no significant difference in the response between patients with a K-Ras gene mutation and wild-type K-Ras gene, who were treated with gefitinib. Therefore, further investigations are required to identify whether or not the K-Ras gene mutation may be used as a response prediction factor in TKI therapy. 
Mutations of the BRAF protein serine/threonine kinase gene have largely been studied in melanomas. Analysis of the BRAF sequence from 127 primary human lung adenocarcinomas revealed mutations in two tumor specimens, one in exon $11(\mathrm{G} 465 \mathrm{~V})$, and a second in exon 15 (L596R) (16). It is clear that the mutation rate of the BRAF gene in lung cancer is relatively low and that BRAF gene non-mutation in our study may be due to the small number of investigated cases.

PIK3CA is significant in the carcinogenesis of the human lung. Mutations in PIK3CA were found in five (3.6\%) of the 139 available patients. PIK3CA has two common mutants, K545E and H1047R (17). In our investigation, PIK3CA mutations were found in 3 patients (10\%), including the PIK3CA codon 542, 545 and 1047 mutational sites, which was consistent with results reported by Okudela et al (17).

ERCC1 plays a critical role in the repair of lesions in DNA induced by cisplatin. Patients with completely resected NSCLC and ERCC1-negative tumors appear to benefit from adjuvant cisplatin-based chemotherapy, unlike patients with ERCC1-positive tumors. However, in patients who did not receive adjuvant chemotherapy, those with ERCC1-positive tumors survived longer than patients with ERCC1-negative tumors (18). ERCC1 is a promising biomarker in advanced NSCLC. Median OS in ERCC1-negative individuals is better than that of the ERCC1-positive population. Patientreported quality of life (QOL) deteriorated significantly among survival-favorable ERCC1-negative patients, possibly due to increased toxicity, particularly in patients with adenocarcinomas. The median OS among patients with adenocarcinomas $(\mathrm{n}=122)$ was 15.2 and 8.3 months, respectively $(p=0.007)$ (4). In the present study, the expression of ERCC1 in females with adenocarcinoma was found to be higher than that in males with squamous cell carcinoma. Whether chemotherapy without platinum can be considered as a regimen for LA-NSCLC patients with positive ERCC1 remains to be investigated.

TUBB3 has been identified as a marker of drug resistance in human cancer. Expression levels of TUBB3 in tumor cells are predictive of response to therapy in patients with NSCLC receiving paclitaxel-based chemotherapy. Patients receiving paclitaxel, whose tumors expressed low levels of TUBB3 isotype, had a better response rate, longer PFS and longer OS (5). In our study, the expression of TUBB3 mRNA in adenocarcinoma patients was much higher than that in squamous cell carcinoma patients. Paclitaxel category-containing chemotherapy considered as a regimen for LA-NSCLC patients with a low expression of TUBB3, also required our attention. At present, a stage II clinical trial (RT0902), which aimed to investigate the effect of concurrent chemoradiotherapy with a paclitaxel-containing regimen, combined with endostar, in treating LA-NSCLC is being carried out by our scientific research group. In this clinical trial, molecular markers such as $\beta$-tubulin and ERCC1 have also been detected.

Pemetrexed is active in patients with non-squamous cell advanced NSCLC, whereas no benefit is observed in patients with squamous-cell histology, possibly as a result of a variable expression of intratumoral TYMS. These are important steps towards individualization of anticancer treatment in patients with advanced NSCLC (6). Consistent with the results of Ceppi et al (19), the results of this study showed that the expression of TYMS mRNA in adenocarcinoma patients was much lower than the expression levels in squamous cell carcinoma patients. The results of the CALGB30407 trial revealed that the OS time of squamous cell carcinoma and non-squamous cell carcinoma patients was 18 and 22 months, respectively. The OS rate of 18 months in squamous cell carcinoma and non-squamous cell carcinoma patients was 48 and 56\%, respectively (20). The results reported in our study also highlight that the PFS was longer in adenocarcinoma patients than in squamous cell carcinoma patients ( 12 vs. 8 months, $\mathrm{p}=0.068)(21)$. The tolerance of the platin-pemetrexed regimen was superior to the third-line platin-containing regimens, which was beneficial for the synchronic radiotherapy treatment.

In conclusion, NSCLC patients should be selected for gefitinib based on the mutation of EGFR, not just based on clinical factors. The incidence of mutations of exons 19 and 21 of the EGFR gene was higher in female patients and non-smokers with NSCLC, than in males and smokers, and it is positively correlated with EGFR mRNA expression. The rate of K-ras and PIK3CA gene mutations were both $10 \%$. No mutations were detected in the BRAF gene. The expression of ERCC1 mRNA and TUBB3 mRNA in adenocarcinoma patients was much higher than that in squamous cell carcinoma patients, and the expression of TYMS mRNA in adenocarcinoma was lower. Improved treatment regimen options may include the use of erlotinib or gefitinib, combined with synchronic pectoral radiotherapy (used for patients with mutations of EGFR exons 19 and 21), platinum-pemetrexed combined with synchronic pectoral radiotherapy (used for adenocarcinoma patients), chemotherapy without platinum (used for patients with positive ERCC1) and paclitaxel category-containing chemotherapy (used for patients with low expression of TUBB3 in LA-NSCLC). However, all of these regimens require further clinical trials to support their efficacy. Detection of these treatment response-related gene mutations or their expression in NSCLC with stage IIIA may be beneficial for individual therapy of LA-NSCLC with chemoradiotherapy, or TKIs combined with synchronic pectoral radiotherapy.

\section{Acknowledgements}

This study was funded by the WKJ2009-2-018, Ministry of Public Health Science Research Fund - Zhejiang Province Medical Science Major Technology Fund Project. Professor Shenglin Ma was awarded the Health High-Level Innovative Talent award in the region of Zhejiang Province.

\section{References}

1. O'Rourke N and Macbeth F: Is concurrent chemoradiation the standard of care for locally advanced non-small cell lung cancer? A review of guidelines and evidence. Clin Oncol 22: 347-355, 2010.

2. Douillard JY, Shepherd FA, Hirsh V, et al: Molecular predictors of outcome with gefitinib and docetaxel in previously treated non-small-cell lung cancer: data from the randomized phase III INTEREST trial. J Clin Oncol 28: 744-752, 2010.

3. Mok TS, Wu YL, Thongprasert S, et al: Gefitinib or carboplatin-paclitaxel in pulmonary adenocarcinoma. N Engl J Med 361: 947-957, 2009.

4. Vilmar A, Santoni-Rugiu E and Sørensen JB: ERCC1, toxicity and quality of life in advanced NSCLC patients randomized in a large multicentre phase III trial. Eur J Cancer 46: 1554-1562, 2010 . 
5. Sève $\mathrm{P}$, Mackey J, Isaac $\mathrm{S}$, et al: Class III beta-tubulin expression in tumor cells predicts response and outcome in patients with non-small cell lung cancer receiving paclitaxel. Mol Cancer Ther 4: 2001-2007, 2005.

6. Joerger M, Omlin A, Cerny T, et al: The role of pemetrexed in advanced non small-cell lung cancer: special focus on pharmacology and mechanism of action. Curr Drug Target 11:37-47, 2010.

7. Rosell R, Moran T, Queralt C, et al: Screening for epidermal growth factor receptor mutations in lung cancer. N Engl J Med 361: 958-967, 2009.

8. Wu YL, Zhong WZ, Li LY, et al: Epidermal growth factor receptor mutations and their correlation with gefitinib therapy in patients with non-small cell lung cancer: a meta-analysis based on updated individual patient data from six medical centers in mainland China. J Thorac Oncol 2: 430-439, 2007.

9. Dahabreh IJ, Linardou H, Siannis F, et al: Somatic EGFR mutation and gene copy gain as predictive biomarkers for response to tyrosine kinase inhibitors in non-small cell lung cancer. Clin Cancer Res 16: 291-303, 2010.

10. Martinez E, Martinez M, Viñolas N, et al: Feasibility and tolerability of the addition of erlotinib to 3D thoracic radiotherapy (RT) in patients (p) with unresectable NSCLC: a prospective randomized phase II study. J Clin Oncol 26 (Suppl): 7563, 2008.

11. Slebos RJ, Kibbelaar RE, Dalesio ON, et al: K-ras oncogene activation as a prognostic marker in adenocarcinoma of the lung. N Engl J Med 323: 561-565, 1990.

12. Mascaux C, Iannino N, Martin B, et al: The role of RAS oncogene in survival of patients with lung cancer: a systematic review of the literature with meta-analysis. Br J Cancer 92: 131-139, 2005.

13. Winton T, Livingston R, Johnson D, et al: Vinorelbine plus cisplatin vs. observation in resected non-small-cell lung cancer. N Engl J Med 352: 2589-2597, 2005.
14. Gautschi O, Huegli B, Ziegler A, et al: Origin and prognostic value of circulating KRAS mutations in lung cancer patients. Cancer Lett 254: 265-273, 2007.

15. Jackman DM, Miller VA, Cioffredi LA, et al: Impact of epidermal growth factor receptor and KRAS mutations on clinical outcomes in previously untreated non-small cell lung cancer patients: results of an online tumor registry of clinical trials. Clin Cancer Res 15: 5267-5273, 2009

16. Naoki K, Chen TH, Richards WG, et al: Missense mutations of the BRAF gene in human lung adenocarcinoma. Cancer Res 62: 7001-7003, 2002

17. Okudela K, Suzuki M, Kageyama S, et al: PIK3CA mutation and amplification in human lung cancer. Pathol Int 57: 664-671, 2007.

18. Olaussen KA, Dunant A, Fouret P, et al: DNA repair by ERCC1 in non-small-cell lung cancer and cisplatin-based adjuvant chemotherapy. N Engl J Med 355: 983-991, 2006.

19. Ceppi P, Volante M, Saviozzi S, et al: Squamous cell carcinoma of the lung compared with other histotypes shows higher messenger RNA and protein levels for thymidylate synthase. Cancer 107: 1589-1596, 2006

20. Govindan R, Bogart J, Wang X, et al: Phase II study of pemetrexed, carboplatin, and thoracic radiation with or without cetuximab in patients with locally advanced unresectable non-small cell lung cancer: CALGB 30407. J Clin Oncol 27 (Suppl): 7505, 2009.

21. Shenglin M, Yaping X, Yongling J, et al: Concomitant pemetrexed/carboplatin chemotherapy and 3D conformal radiotherapy followed by pemetrexed/carboplatin consolidation chemotherapy for locally advanced non-small cell lung cancer: preliminary results of a phase II study. J Clin Oncol 28 (Suppl): 7092, 2010. 